\title{
Electronic structure of $\mathrm{LaNiO}_{3-x}$ thin films studied by x-ray photoelectron spectroscopy and density functional theory
}

\author{
Š. Masys, ${ }^{1, *}$ S. Mickevičius, ${ }^{2, \dagger}$ S. Grebinskij, ${ }^{2}$ and V. Jonauskas ${ }^{1, \ddagger}$ \\ ${ }^{1}$ Institute of Theoretical Physics and Astronomy, Vilnius University, A. Goštauto Street 12, LT-01108 Vilnius, Lithuania \\ ${ }^{2}$ State Scientific Research Institute, Center for Physical Sciences and Technology, A. Goštauto Street 11, LT-01108 Vilnius, Lithuania
}

(Received 18 June 2010; published 21 October 2010)

\begin{abstract}
We present the experimental and theoretical study of x-ray photoelectron spectroscopy spectrum of $\mathrm{LaNiO}_{3-x}$ thin films deposited onto a (100)-plane oriented $\mathrm{NdGaO}_{3}$ substrate by applying a reactive dc magnetron sputtering technique. The crystal calculations carried out within pure density functional theory (DFT) approximation properly reproduce the delocalized valence band showing the suitability of homogeneous electron gas model, whereas hybrid DFT/Hartree-Fock (HF) calculations work out in the core region indicating the localized nature of core electrons. The significance of spin-orbit coupling to the electronic structure of $\mathrm{LaNiO}_{3}$ is revealed by relativistic molecular HF study allowing to theoretically identify the doublet splitting of La $5 p$. Electron-density difference map indicates a different nature of $\mathrm{La}-\mathrm{O}$ and $\mathrm{Ni}-\mathrm{O}$ chemical bonds in $\mathrm{LaNiO}_{3}$ while the Mulliken population analysis adds information about the charge distributions and overlap populations between separate atoms.
\end{abstract}

DOI: 10.1103/PhysRevB.82.165120

PACS number(s): 71.20.-b, 79.60.-i, 71.27.+a

\section{INTRODUCTION}

$\mathrm{LaNiO}_{3}$ is a highly conductive oxide with a perovskitetype structure, exhibiting metallic behavior over a wide temperature range. ${ }^{1,2}$ This compound has attracted significant theoretical and experimental research efforts due to its tremendous potential to be used in developing colossal magnetoresistance materials, ${ }^{3}$ solid oxide fuel cells, ${ }^{4}$ hightemperature superconductors, ${ }^{5}$ and ferroelectric thin-film devices. ${ }^{6}$

The perovskite structure of $\mathrm{LaNiO}_{3}$ is rhombohedrally distorted with a pseudocubic lattice parameter of $3.84 \AA$ (space group $R \overline{3} c$ ), ${ }^{7}$ which matches well with the unit cells of several common superconductors and ferroelectrics (e.g., $\mathrm{YBa}_{2} \mathrm{Cu}_{3} \mathrm{O}_{7-z}, \mathrm{PbTiO}_{3}$, and $\mathrm{BaTiO}_{3}$ ) allowing epitaxial growth of these perovskites on $\mathrm{LaNiO}_{3}$ substrate. Furthermore, $\mathrm{LaNiO}_{3}$ is conductive with electrical resistivity of only a few hundred microhm centimeter at room temperature and shows metalliclike temperature dependence. ${ }^{8}$

The main aim of our study presented in this paper is to investigate the electronic structure of $\mathrm{LaNiO}_{3-x}$ thin films by applying reliable experimental and theoretical tools. Previously reported $\mathrm{LaNiO}_{3}$ thin films were prepared by such physical vapor deposition methods as molecular-beam epitaxy, ${ }^{9}$ pulsed laser deposition, ${ }^{10}$ rf magnetron sputtering, ${ }^{11}$ and spin coating. ${ }^{12}$ In this paper, $\mathrm{LaNiO}_{3-x}$ films are fabricated on $\mathrm{NdGaO}_{3}$ substrates by a reactive dc magnetron sputtering system and demonstrate an excellent in-plane orientation. ${ }^{13}$ The x-ray photoelectron spectroscopy (XPS) study indicates that the surface $\mathrm{La} / \mathrm{Ni}$ concentration ratio is close to the one of stoichiometric volume. ${ }^{14}$ As for theoretical analysis, we pay considerable attention to the core band region since it requires relativistic treatment, namely, inclusion of spin-orbit interaction, which, to the best of our knowledge, has not yet been applied in the investigation of $\mathrm{LaNiO}_{3}$. Moreover, the electronic-structure calculations of $\mathrm{LaNiO}_{3}$ reported so far were performed using plane-wave and muffin-tin orbital methods within local-density,DFT $+U$, and $U+\mathrm{GW}$ approximations (see, e.g., Refs. 15-19). In this paper, a theoretical investigation is based on more flexible linear combination of atomic orbitals (LCAOs) approach allowing to have a better insight on chemical bonding in $\mathrm{LaNiO}_{3}$. In addition, the band structure analysis permits to assess the metallic character of $\mathrm{LaNiO}_{3}$ and the Mulliken population analysis, not presented in previous works, demonstrates the charge distributions and overlap populations between $\mathrm{La}, \mathrm{Ni}$, and $\mathrm{O}$ atoms.

\section{EXPERIMENT}

Thin $\mathrm{LaNiO}_{3-x}$ films were deposited onto monocrystalline (100)-plane oriented $\mathrm{NdGaO}_{3}$ substrate by using a reactive dc magnetron sputtering technique. The ceramic $\mathrm{LaNiO}_{3}$ target ( $25 \mathrm{~mm}$ in diameter and $2.5 \mathrm{~mm}$ in thickness) was prepared by pressing at $5 \times 10^{8} \mathrm{~Pa}$ and after sintering in air at $1000{ }^{\circ} \mathrm{C}$ for $10 \mathrm{~h} \mathrm{La}_{2} \mathrm{O}_{3}$ and $\mathrm{NiO}(99.99 \%$ purity from Aldrich-Chemie) powders in the stoichiometric ratio. The sputtering was performed in $\mathrm{Ar}$ and $\mathrm{O}_{2}$ mixture (20:1) at pressure of about $15 \mathrm{~Pa}$. To prevent the film bombardment by high-energy ions during deposition, $\mathrm{NdGaO}_{3}$ substrates were positioned in off-axis configuration at a distance of $15 \mathrm{~mm}$ from the symmetry axis of the discharge and $20 \mathrm{~mm}$ over the target plane. The substrate temperature was $\sim 750{ }^{\circ} \mathrm{C}$. Under these conditions, the deposition rate was $25 \mathrm{~nm} / \mathrm{h}$, and the resultant thickness of $\mathrm{LaNiO}_{3-x}$ film was about $0.1 \mu \mathrm{m}$. Films demonstrate an excellent in-plane orientation ${ }^{13}$ and the surface $\mathrm{La} / \mathrm{Ni}$ ratio is close to the bulk stoichiometric value. ${ }^{14}$

The XPS data was recorded with the tunable high-energy $\mathrm{x}$-ray photoelectron spectrometer at the $\mathrm{x}$-ray wiggler beamline BW2 of HASYLAB (Hamburg). The phonon flux from the Si (111) double-crystal monochromator typically amounts to $5 \times 10^{12} \mathrm{~s}^{-1}$ on the sample. The photoelectrons were measured using a hemispherical analyzer with parallel detection capability (SCIENTA SES-200). Excitation energy $h \nu=3000 \mathrm{eV}$ was chosen where the kinetic energy of photoelectrons from $\mathrm{La}$ and $\mathrm{Ni}$ core levels does not overlap with 
any of Auger electron emission peaks. The total energy resolution at this photon energy was $0.6 \mathrm{eV}$. The binding energy scale was calibrated using the photoemission from gold reference sample. The adventitious carbon $\mathrm{C} 1 s$ line was used for the fine correction of the charging effects, supposing its binding energy should be equal to $284.6 \mathrm{eV}$. After Shirley background subtraction, the complex photoelectron spectra were decomposed into separate peaks by specifying the peak position binding energy, area, width, and Gaussian/ Lorentzian $(\mathrm{G} / \mathrm{L})$ ratio. Because any mathematical operation (such as data smoothing) on the raw data would distort the original physical picture, no such treatment was permitted in the quantitative data analysis. For core level and valenceband region outer peaks fitting procedure provides $\mathrm{G} / \mathrm{L}$ ratio close to 1 , indicating that phonon and instrumental broadening dominates in peak energy distribution. ${ }^{20}$ Therefore, it is logical to select a Gaussian function to represent the energy distribution of inner valence band peaks. The second derivate plots method has been used to determine hidden peaks position in valence-band region.

\section{COMPUTATIONAL METHOD}

In this paper we assume that $\mathrm{LaNiO}_{3}$ crystal has a tetragonally distorted structure with lattice constants $(a=b=3.905 \AA, c=3.811 \AA)$ taken from Ref. 15. Our major calculations were carried out using CRYSTAL06 computer code, ${ }^{21}$ which is designed for Hartree-Fock (HF) and KohnSham density-functional theory (DFT) calculations on crystalline materials, employing LCAO basis set. In order to take into account scalar-relativistic effects for the inner shell electrons of heavy La atom, we used small core Hay-Wadt ${ }^{22}$ effective core pseudopotential (ECP). The valence basis set for La applied with this ECP was taken from the original Hay and Wadt's paper without any modification (electronic configuration $5 s^{2} 5 p^{6} 5 d^{1} 6 s^{2}$ ) since in the literature there is a lack of systematically optimized valence basis set for La suitable to perform crystal calculations. Numerical problems usually caused by too diffuse valence functions, as mentioned in Refs. 23-25, have been worked out by extending the $\mathrm{LaNiO}_{3}$ unit cell from 5 to 20 atoms (4 f.u., space group $I 4 / \mathrm{mcm}$ ). For Ni, we also used small core Hay-Wadt ECP combining it with valence basis set taken from CRYSTAL website ${ }^{26}$ (electronic configuration $3 s^{2} 3 p^{6} 3 d^{8} 4 s^{2}$ ). For $\mathrm{O}$, two all-electron basis sets were adopted from the nickel mono-oxide ${ }^{27}$ and hexagonal hydroxyapatite ${ }^{28}$ studies.

We have based our calculations on several theoretical methods: HF, DFT with the generalized gradient approximation (GGA), and hybrid functionals. Within the DFT-GGA framework, calculations were performed using Becke exchange functional ${ }^{29}$ combined with a correlation functional suggested by Lee, Yang, and Parr ${ }^{30}$ (BLYP), and exchangecorrelation functionals proposed by Perdew, Burke, and Ernzerhof $^{31}$ (PBE). Hybrid functional method involved hybrid B3LYP (Ref. 32) and PBE0 (Ref. 33) functionals, which can be given by

$$
E_{X C}^{\mathrm{B} 3 \mathrm{LYP}}=E_{X C}^{\mathrm{LSDA}}+a\left(E_{X}^{\mathrm{HF}}-E_{X}^{\mathrm{LSDA}}\right)+b \Delta E_{X}^{\mathrm{B}}+c \Delta E_{C}^{\mathrm{LYP}},
$$

$$
E_{X C}^{\mathrm{PBE} 0}=E_{X C}^{\mathrm{PBE}}+\frac{1}{4}\left(E_{X}^{\mathrm{HF}}-E_{X}^{\mathrm{PBE}}\right) .
$$

In Eq. (1), $E_{X C}^{\mathrm{LSDA}}$ is the local spin-density approximation (LSDA) for the exchange-correlation energy, $\Delta E_{X}^{\mathrm{B}}$ and $\Delta E_{C}^{\mathrm{LYP}}$ correspond to the Becke's and Lee-Yang-Parr's gradient corrections for the local exchange and correlation energies, respectively. The $a=0.2, b=0.72$, and $c=0.81$ parameters have been optimized to fit the experimental data. In Eq. (2), $E_{X C}^{\mathrm{PBE}}$ expresses the Perdew-Burke-Ernzerhof's exchangecorrelation energy, and in both B3LYP and PBE0 methods, $E_{X}^{\mathrm{HF}}$ denotes the exact $\mathrm{HF}$ exchange energy.

The shrinking factor of 16 in the Monkhorst-Pack net ${ }^{34}$ corresponding to 349 asymmetric $k$ points in the irreducible Brillouin zone was used for reciprocal-space integration. To improve the self-consistent field convergence, Fock/KohnSham matrix mixing technique (controlled by parameter FMIXING in the code) and Anderson's method ${ }^{35}$ (parameter ANDERSON) were applied. However, the convergence tools worked out within DFT-GGA and hybrid DFT/HF frameworks but failed with HF approximation. To get comparable results of HF method we applied a different basis set for $\mathrm{O}$ by taking it from Ref. 28. The comparative calculations employing this basis within DFT-GGA and hybrid DFT/HF approximations showed that the results are in agreement with those of using basis set taken from Ref. 27, thus allowing to present HF calculations for determination of theoretical tendencies.

Our additional molecular study was performed using DIRAC08 (Ref. 36) and GAUSSIAN03 (Ref. 37) computer codes. Since La is a heavy atom, it is necessary to include not only scalar-relativistic effects, but also a spin-orbit interaction. Unfortunately, spin-orbit interaction is not implicated in CRYSTAL06 code, therefore we investigated influence of it at molecular level by studying $\mathrm{LaNiO}_{3}$ unit cell of 15 atoms with the same lattice constants and tetragonally distorted structure (point group 4/ $\mathrm{mmm}$ ). DIRAC08 is a relativistic $a b$ initio electronic-structure program which computes molecular properties using relativistic quantum chemical methods. For comparison purposes, we performed two HF calculations employing nonrelativistic and two-component relativistic Hamiltonians. The latter one is obtained after Barysz-SadlejSnijders transformation of the Dirac Hamiltonian in the finite basis $\operatorname{set}^{38}$ (parameter BSS 099). We reduced the recommended convergence criterion (norm of the electronic gradient) to $10^{-5}$ because we were only interested in binding energy. The relativistic all-electron double-zeta basis set for $\mathrm{La}$ developed with the Dirac-Coulomb Hamiltonian was taken from Ref. 39. For Ni and O, we confined to standard 3-21G (Ref. 40) and STO-2G (Ref. 41) basis sets, respectively, noticeably reducing computational time. To qualitatively verify results obtained with DIRAC08, we also made nonrelativistic calculations with GAUSSIAN03 using identical basis sets for $\mathrm{Ni}$ and $\mathrm{O}$, and previously mentioned original Hay-Wadt's ECP with its valence basis set for La. Density of states (DOS) data was extracted with the help of GAUSSSUM 2.1 (Ref. 42).

\section{RESULTS AND DISCUSSION}

Experimental $\mathrm{LaNiO}_{3}$ spectrum, shown in Fig. 1, is composed of three main regions. The lowest binding-energy re- 


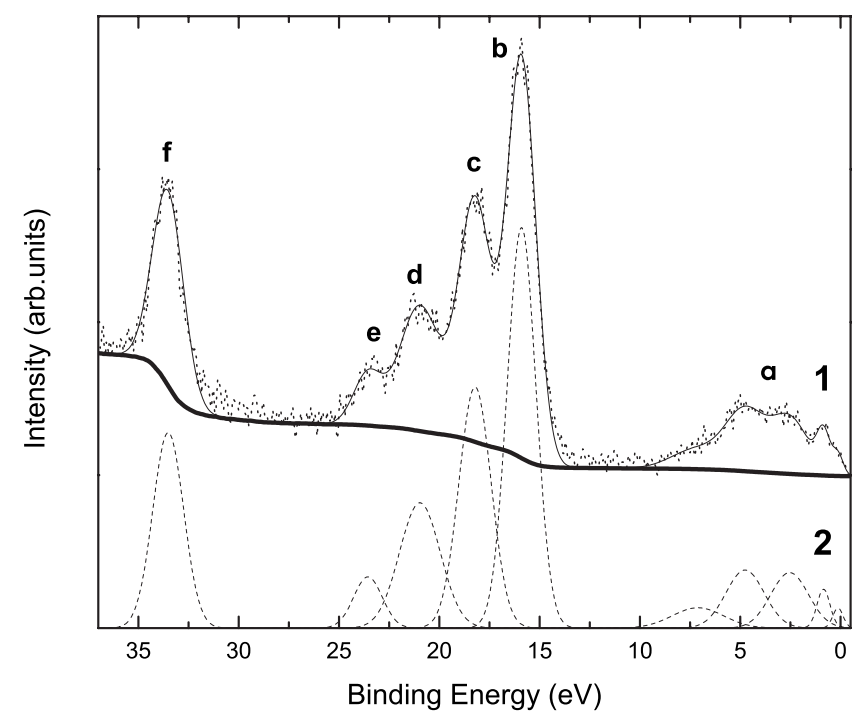

FIG. 1. XPS spectrum of $\mathrm{LaNiO}_{3-x}$ thin film grown onto monocrystalline (100)-plane oriented $\mathrm{NdGaO}_{3}$ substrate: (1) experimental data (dots), Shirley background (thick solid line), and spectrum envelope (thin solid line) and (2) least-squares fitted spectral components (dashed lines).

gion contains a broad peak labeled $a$, above it lie four prominent peaks labeled $b, c, d$, and $e$, and the highest binding energy region contains another sharp peak labeled $f$. The detailed structure of peak $a$ is shown in separate Fig. 2 since valence-band spectrum requires a wider discussion. The total and projected DOS calculated using DFT-GGA, hybrid DFT/HF functionals, and HF method are shown in Figs. 3-5, respectively. Results plotted in Fig. 3 are in a very good agreement with previous plane-wave calculations carried out by Dobin et al. ${ }^{15}$ It can be clearly seen that the valence band is formed by $\mathrm{O} 2 p$ and $\mathrm{Ni} 3 d$ orbitals, whereas the upper

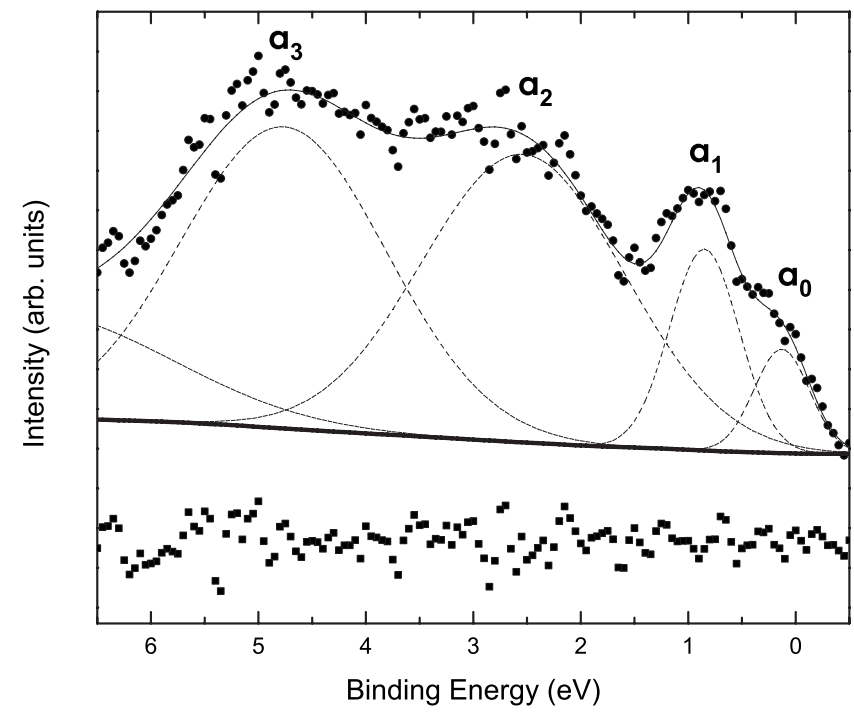

FIG. 2. Detailed XPS spectrum of $\mathrm{LaNiO}_{3-x}$ thin film in valence-band region: experimental data (circle dots), Shirley background (thick solid line), spectrum envelope (thin solid line), leastsquares fitted spectral components (dashed lines), and residual plot (square dots). core band is formed by La $5 p$ and $\mathrm{O} 2 s$ orbitals. The lower core band is essentially dominated by pure La $5 s$ shell. Figures 3 and 4 show a strong hybridization between $\mathrm{O} 2 p$ and $\mathrm{Ni} 3 d$ orbitals, as well as a weak hybridization between La $5 p$ and $\mathrm{O} 2 s$ orbitals, indicating significant and negligible covalent contributions to $\mathrm{Ni}-\mathrm{O}$ and $\mathrm{La}-\mathrm{O}$ chemical bonds, respectively. HF calculations demonstrate a weak $\mathrm{O} 2 p$ and $\mathrm{Ni} 3 d$ hybridization due to lack of correlation interaction playing a particularly important role in strongly correlated nature of $\mathrm{LaNiO}_{3}$.

By comparing calculated and experimental spectra one can notice that Figs. 3-5 exhibit only three peaks in the La $5 p / \mathrm{O} 2 s$ region, whereas in Fig. 1 it can be seen four peaks $b, c, d$, and $e$. Initially, we thought that this discrepancy might be caused by the basis set we applied for $\mathrm{LaNiO}_{3}$. However, our DFT-GGA study exhibited a perfect agreement with the plane-wave based calculations made by Dobin et al. At this point, it is easy to get misled by the conviction that the coincident results of two different basis sets indeed must be correct and therefore to incorrectly identify peaks in the spectrum. It took us awhile to realize the actual reason for disagreement, which proved to be by no means straightforwardly approachable. Since $\mathrm{LaNiO}_{3}$ can be considered as a heavy-element system, we made an assumption that fully relativistic treatment may have a great qualitative improvement on the calculated electronic structure. Thus, to evaluate not only scalar-relativistic effects but also a spin-orbit interaction we carried out a computationally demanding relativistic HF study with DIRAC08. Although this study has been made only at molecular level, La $5 p / \mathrm{O} 2 \mathrm{~s}$ DOS presented in Fig. 6(a) shows a good qualitative agreement with the structure of experimental spectrum, confirming our assumption. The doublet splitting of La $5 p$ indicates that experimental peaks $b$ and $c$ should belong to La $5 p_{3 / 2}$ and La $5 p_{1 / 2}$, respectively. These results are consistent with electron binding energies for La in other solids ${ }^{43}$ as the largest mismatch originates for $5 p_{1 / 2}$ and reaches only $0.35 \mathrm{eV}$ $(2.1 \%)$. Considerable shift in binding energy, seen in Fig. 6(a), is most likely induced due to lack of strong crystal-field and correlation interactions. Corresponding nonrelativistic HF calculations performed with DIRAC08 and GAUSSIAN03 are presented in Figs. 6(b) and 6(c), respectively. As it could be expected, they are in agreement with the structure of DOS calculated with CRYSTAL06 at HF level. Despite the fact that we used different basis sets for La in DIRAC08 and GAUSSIAN03 calculations, Figs. 6(b) and 6(c) match fairy well indicating reliability of results obtained with DIRAC08.

As our theoretical investigation performed with CRYSTAL06 reproduce one La $5 p$ peak instead of two with $J=1 / 2$ and $J=3 / 2$, we labeled it $b / c$ and, for comparison, applied a simple expression which evaluates mean experimental binding energy of La $5 p$,

$$
E_{5 p}=\frac{E_{5 p_{3 / 2}}\left[J_{3 / 2}\right]+E_{5 p_{1 / 2}}\left[J_{1 / 2}\right]}{\left[J_{3 / 2}\right]+\left[J_{1 / 2}\right]},
$$

where $\left[J_{x}\right]=2 x+1$ expresses statistical weight of the energy level. 

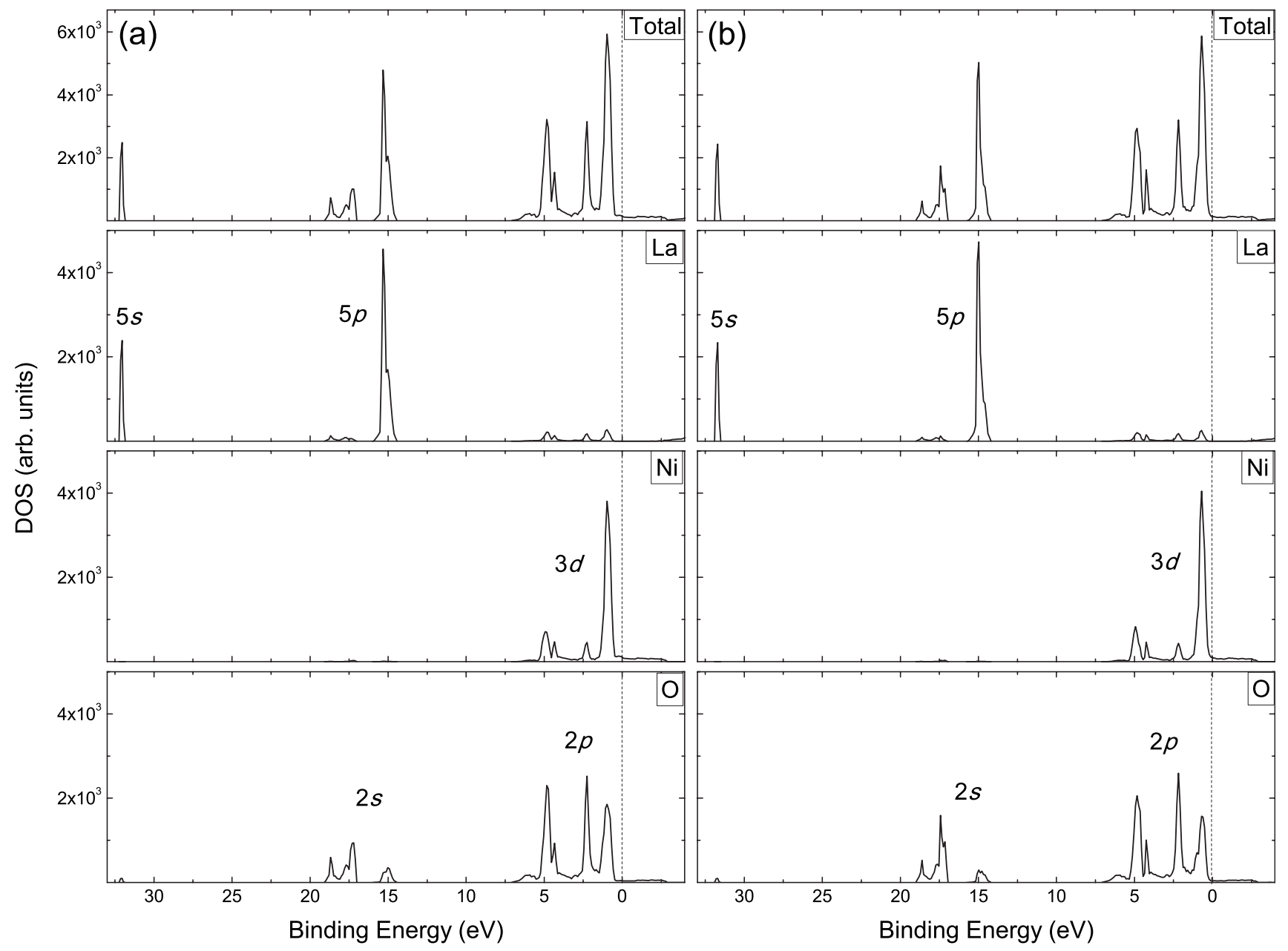

FIG. 3. The total and projected DOS calculated within DFT-GGA framework: (a) BLYP and (b) PBE. The Fermi level is indicated by a vertical dashed line.

Comparison between the binding energies of experimental peaks $d, e, f$, mean value of $b / c$ and corresponding calculated values is presented in Table I. This table demonstrates that DFT-GGA calculations underestimate while HF calculations overestimate binding-energy values. The largest disagreements for DFT-GGA and HF methods reach $4.93 \mathrm{eV}$ $(20.9 \%)$ and $-8.81 \mathrm{eV}(26.3 \%)$, respectively. This tendency is mostly caused by the lack of correlation interaction in HF case, and additional spurious self-interaction in DFT case. The original motivation for applying hybrid DFT/HF scheme in our calculations stems from the need to properly reproduce the strongly correlated nature of $\mathrm{LaNiO}_{3}$. In this type of materials electrons tend to be localized and strongly interacting, thus the self-interaction error (SIE) common for DFT drastically increases, since DFT essentially describes noninteracting electrons moving in an effective self-consistent mean field. The pure HF approximation is SIE free but it omits correlation energy and therefore does not properly represent the physics in strongly correlated systems. However, the incorporation of exact $\mathrm{HF}$ exchange $E_{X}^{\mathrm{HF}}$ part into hybrid functionals partly compensates the SIE and in this way substantially improves the results [e.g., in Table I the largest disagreement reduces to $2.48 \mathrm{eV}(10.5 \%)$ for peak $e$ ]. In other words, hybrid DFT/HF scheme can somewhat serve as a reasonable alternative for more sophisticated SI-corrected DFT or DFT $+U$ methods. As HF mixing parameter reflects the relative importance of a systems independent-particle character, ${ }^{32}$ one may systematically improve the accuracy of results by changing $\mathrm{HF}$ exchange part incorporated into functionals.

Figure 2 presents a detailed structure of experimental spectrum in valence-band region. The binding energies of four peaks labeled $a_{0}, a_{1}, a_{2}$, and $a_{3}$ perfectly agree with those of previous experiment conducted by Horiba et al. ${ }^{16}$ despite the lower total-energy resolution of our measurements. The comparison between the calculated and experimental valence-band spectra reveals that both hybrid DFT/HF and pure HF methods fail to properly reproduce the experiment exhibiting only two shifted peaks and a complex structure lying far from the Fermi level, respectively. On the contrary, pure DFT-GGA calculations show a fairy well agreement. This result can be explained by the fact that DFTGGA method is perfectly consistent with homogeneous electron gas model. Since $\mathrm{LaNiO}_{3}$ exhibits strong metallic character (see further text and Fig. 8), electrons in valence band can be considered being delocalized through the whole solid. The delocalized electrons move fast and experience mainly the DFT-GGA mean-field potential, ${ }^{44}$ thus allowing the ho- 

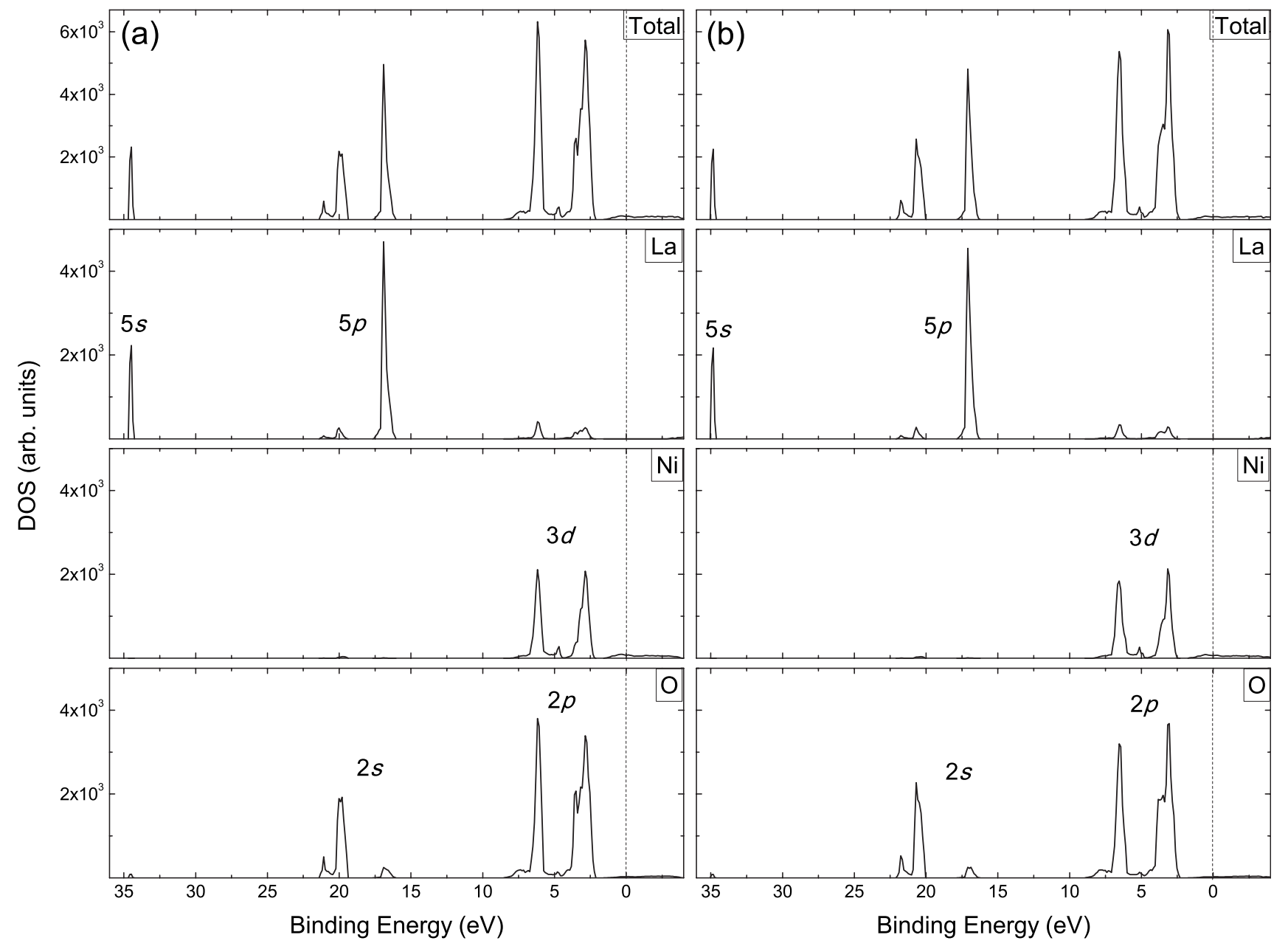

FIG. 4. The total and projected DOS calculated within hybrid DFT/HF framework: (a) B3LYP and (b) PBE0. The Fermi level is indicated by a vertical dashed line.

mogeneous electron gas model to properly reproduce the nature of metallic character. As hybrid DFT/HF functionals incorporate some part of exact $\mathrm{HF}$ exchange $E_{X}^{\mathrm{HF}}$ to

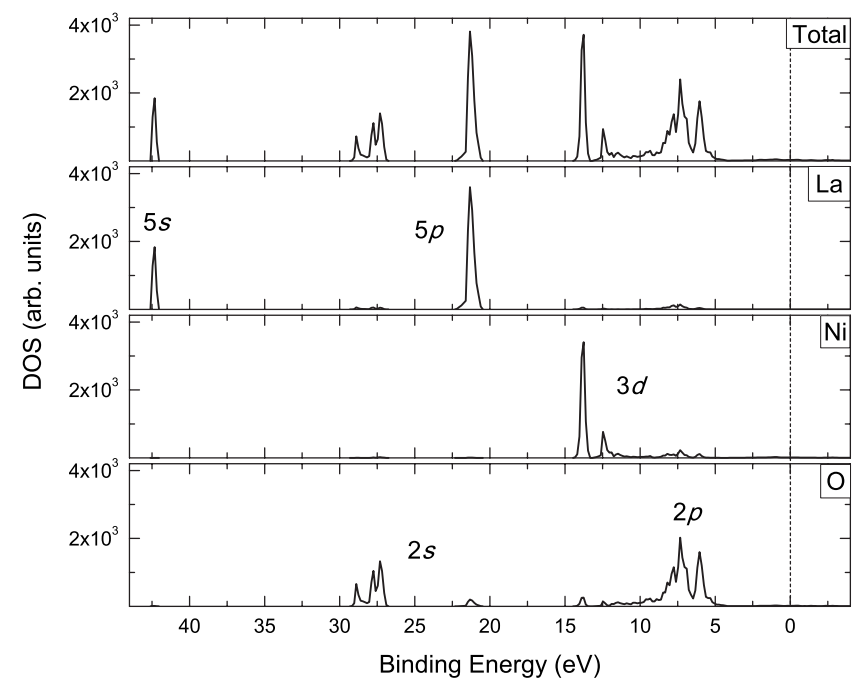

FIG. 5. The total and projected DOS calculated within HF framework. The Fermi level is indicated by a vertical dashed line. compensate the SIE, they properly behave in regions where electrons preserve their strongly localized nature. Table I demonstrates that hybrid functionals give the closest results to the experiment in the core region while Fig. 4 indicates that valence-band region gets rather overcorrected. Pure DFT-GGA functionals due to spurious self-interaction underestimate binding energies in the core region but correctly reproduce the delocalized valence band. Thus, by applying pure DFT approximation and hybrid DFT/HF scheme for different regions of spectrum we are able to capture the essential physics in $\mathrm{LaNiO}_{3}$. HF calculations suffer from the lack of correlation interaction and therefore give the poorest results.

Comparison between the binding energies of experimental peaks $a_{1}, a_{2}$, and $a_{3}$ and corresponding calculated values within DFT-GGA method is listed in Table II. We do not present the results of hybrid $\mathrm{DFT} / \mathrm{HF}$ and $\mathrm{HF}$ calculations because as it was already mentioned both of these methods fail to attain the homogeneous electron gas limit. The largest discrepancies of only $0.27 \mathrm{eV}$ and 0.39 for BLYP and PBE, respectively, show a high reliability of our calculations. However, we did not reproduce any structure that would correspond to small peak $a_{0}$ lying in the vicinity of the Fermi level. This valence-band region might be very sensitive to 

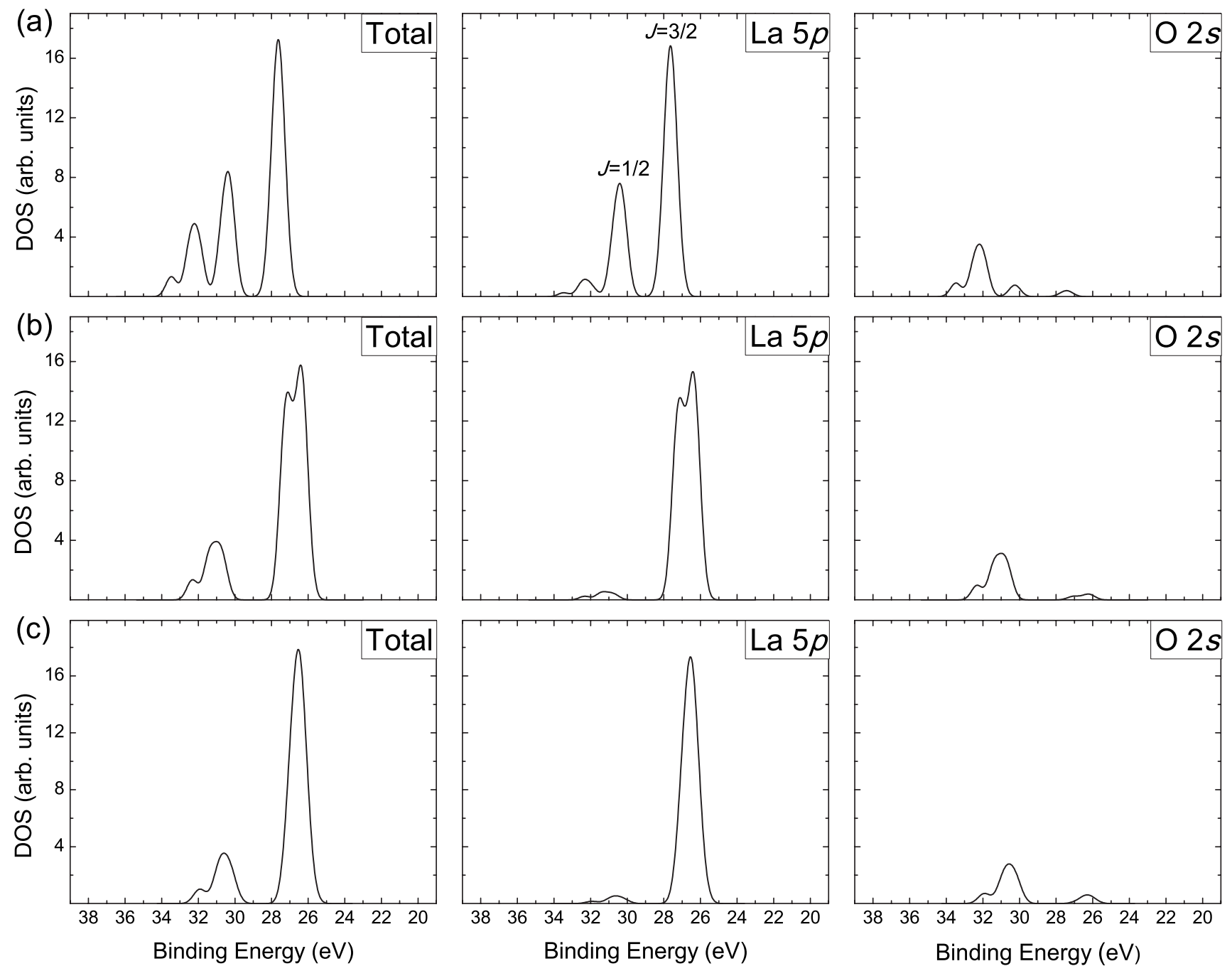

FIG. 6. The total and projected DOS of La $5 p / \mathrm{O} 2 s$ region calculated at molecular HF level using: (a) DIRAC08 with relativistic Hamiltonian, (b) DIRAC08 with nonrelativistic Hamiltonian, and (c) GAUSSIAN03 with nonrelativistic Hamiltonian.

the choice of basis set and requires a further study.

The electron-density difference map, obtained by subtracting the superposition of spherical atomic charge distributions from the crystal electron density in the crystallographic plane (110), is shown in Fig. 7. It should be stressed that this type of analysis was not reported in previous studies

TABLE I. Calculated binding energies $E(\mathrm{eV})$ and their deviations from experimental values $\Delta E(\mathrm{eV})$ in the core region.

\begin{tabular}{lcrrrrrr}
\hline \hline Peak & Quantity & BLYP & PBE & B3LYP & PBE0 & HF & Experiment \\
\hline$b / c$ & $E$ & 15.33 & 15.02 & 16.90 & 17.09 & 21.32 & 16.67 \\
& $\Delta E$ & 1.34 & 1.65 & -0.23 & -0.42 & -4.65 & \\
$d$ & $E$ & 17.44 & 17.21 & 20.01 & 20.68 & 27.32 & 20.97 \\
& $\Delta E$ & 3.53 & 3.76 & 0.96 & 0.29 & -6.35 & \\
$e$ & $E$ & 18.63 & 18.67 & 21.08 & 21.78 & 28.89 & 23.56 \\
& $\Delta E$ & 4.93 & 4.89 & 2.48 & 1.78 & -5.33 & \\
$f$ & $E$ & 32.05 & 31.71 & 34.48 & 34.83 & 42.32 & 33.51 \\
& $\Delta E$ & 1.46 & 1.80 & -0.97 & -1.32 & -8.81 & \\
\hline \hline
\end{tabular}

based on plane-wave or muffin-tin orbital approximations. We present the results calculated using BLYP functional to reduce the amount of figures in this paper since all the other methods exhibit practically the same picture. As it can be seen in Fig. 7, the electron density significantly increases at $\mathrm{O}$ atom in the $\mathrm{La}-\mathrm{O}$ bond direction, whereas $\mathrm{La}$ atom is surrounded by negative, zero value, and positive isolines, implying that covalent contribution to the La-O bond is small. The decrease in electron density at both $\mathrm{O}$ and $\mathrm{Ni}$

TABLE II. Calculated binding energies $E(\mathrm{eV})$ and their deviations from experimental values $\Delta E(\mathrm{eV})$ in the valence region.

\begin{tabular}{lcrcc}
\hline \hline Peak & Quantity & BLYP & PBE & Experiment \\
\hline$a_{1}$ & $E$ & 0.99 & 0.70 & 0.85 \\
& $\Delta E$ & -0.14 & 0.15 & \\
$a_{2}$ & $E$ & 2.27 & 2.15 & 2.54 \\
& $\Delta E$ & 0.27 & 0.39 & \\
$a_{3}$ & $E$ & 4.85 & 4.80 & 4.76 \\
& $\Delta E$ & -0.09 & -0.04 & \\
\hline \hline
\end{tabular}




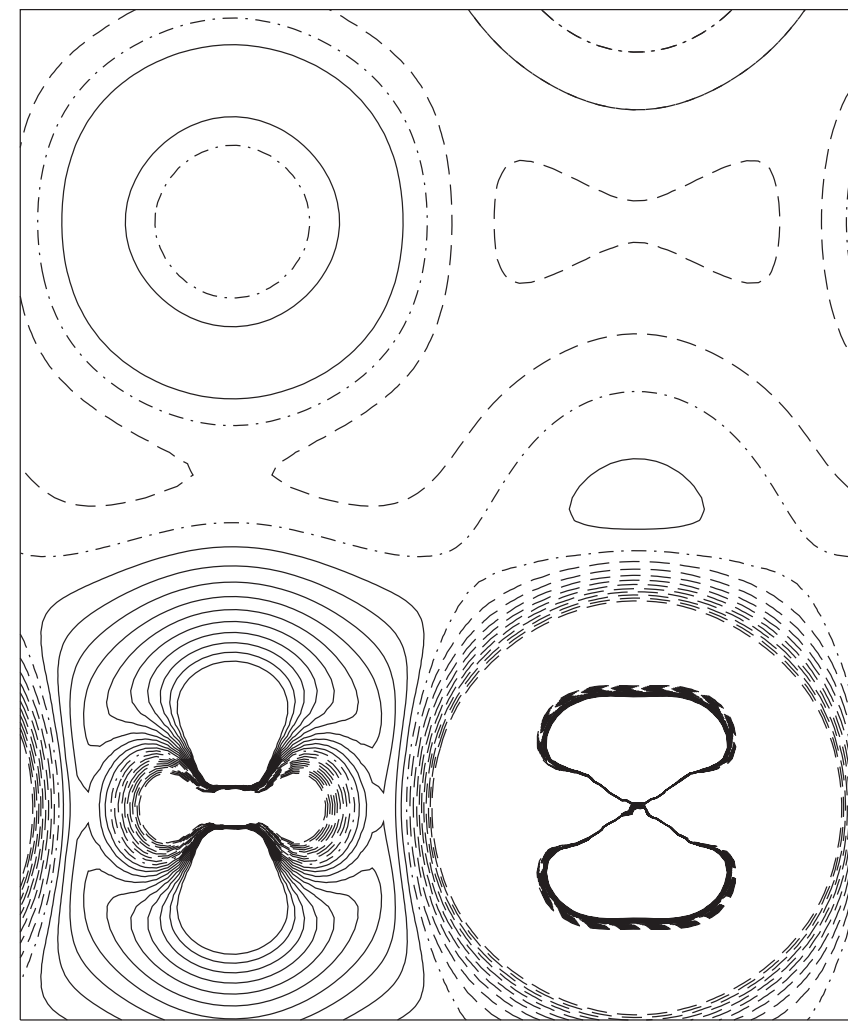

FIG. 7. The electron-density difference map in the crystallographic plane (100) calculated using BLYP functional. Dashed, dotted-dashed, and continuous isolines represent negative, zero value, and positive differences of density, respectively. The isolines are drawn from -0.025 to $0.025 e /$ bohr $^{3}$ with a step of $0.0025 \mathrm{e} / \mathrm{bohr}^{3}$. La, O, and $\mathrm{Ni}$ atoms are located at the top left, bottom left, and bottom right sides of the map, respectively.

atoms gradually transforming to a tight concentration of positive isolines within the $\mathrm{Ni}-\mathrm{O}$ bond explicitly shows a substantial covalent part in the Ni-O bond. This pictorial representation confirms our previous remarks made on chemical bonding in $\mathrm{LaNiO}_{3}$, as predominantly ionic nature of La-O bond is also indicated by much weaker hybridization than that of Ni-O atoms, apparently seen in Figs. 3-5.

The Mulliken population analysis, directly available using LCAO approach, is shown in Table III. It demonstrates that our results differ from formal ionic picture of $\mathrm{La}^{3+}, \mathrm{Ni}^{3+}$, and $\mathrm{O}^{2-}$. One can notice that the atomic charges increase as the part of the exact exchange interaction included in

TABLE III. Mulliken atomic charges $Q(e)$ and overlap populations $O P\left(10^{-3} e\right)$.

\begin{tabular}{lccccc}
\hline \hline Quantity & BLYP & B3LYP & PBE & PBE0 & HF \\
\hline$Q(\mathrm{La})$ & 1.610 & 1.761 & 1.603 & 1.785 & 2.086 \\
$Q(\mathrm{Ni})$ & 1.655 & 1.697 & 1.662 & 1.718 & 1.962 \\
$Q(\mathrm{O})$ & -1.089 & -1.156 & -1.086 & -1.175 & -1.294 \\
$O P(\mathrm{La}-\mathrm{O})$ & 36 & 28 & 38 & 26 & 46 \\
$O P(\mathrm{Ni}-\mathrm{O})$ & 60 & 56 & 58 & 56 & 66 \\
$O P(\mathrm{O}-\mathrm{O})$ & -24 & -27 & -24 & -27 & -6 \\
\hline \hline
\end{tabular}

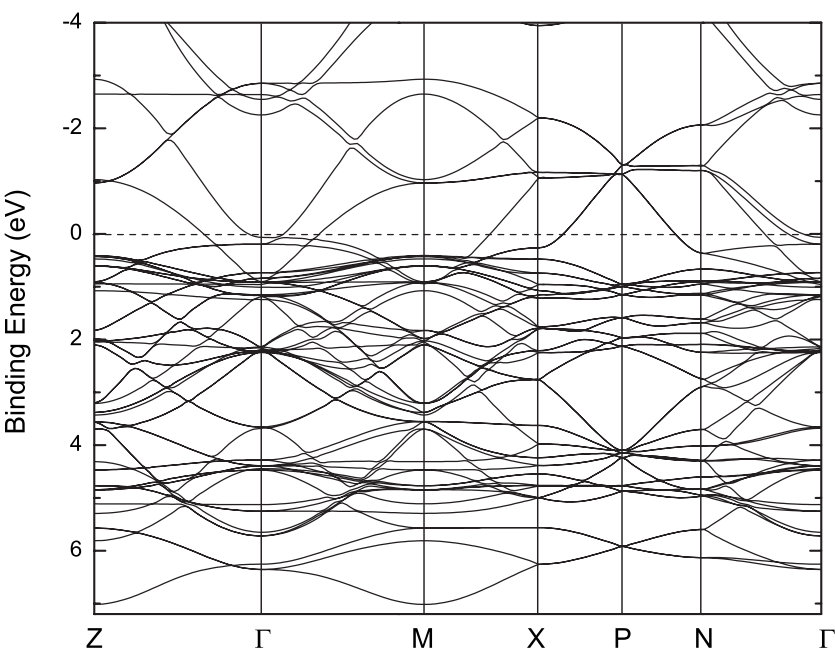

FIG. 8. The band structure of the valence and lower conduction bands calculated using BLYP functional. The Fermi level is indicated by a horizontal dashed line.

calculations gets larger: e.g., from $Q(\mathrm{La})_{\mathrm{BLYP}}=1.610 e$ to $Q(\mathrm{La})_{\mathrm{B} 3 \mathrm{LYP}}=1.761 e$ and eventually $Q(\mathrm{La})_{\mathrm{HF}}=2.086 e$. Nevertheless, calculated charges and overlap populations indicate a significant covalent part in chemical bonding for $\mathrm{Ni}-\mathrm{O}$, as well as La-O atoms. Since Mulliken population analysis is strongly basis set dependent, surprisingly large overlap populations of La-O atoms imply that basis set we applied for La contains too diffuse functions, also resulting in a considerable charge deviation from formal +3 . Thus, in order to distinguish an appropriate part of covalent contribution to $\mathrm{La}-\mathrm{O}$ bond, it is necessary to systematically optimize basis set for La.

The calculated band structure is given in Fig. 8. Although it is a well-known fact that neither pure DFT-GGA nor pure HF calculations correctly reproduce the band structure for semiconductors and insulators, it is not the case for metals because DFT-GGA method is able to represent the delocalized nature of electrons in the valence band. Since most of all we are interested in the band structure located near the Fermi level, Fig. 8 presents the results obtained with BLYP functional. As the highest valence and the lowest conduction bands intercross each other, a zero band gap occurs indicating a strong metallic character of $\mathrm{LaNiO}_{3}$. From Fig. 9, presenting projected DOS in the vicinity of the Fermi level, it can be seen that the top valence and bottom conduction bands are composed of dominant Ni $e_{g}$ doublet mixed with some part of $\mathrm{O} p$. These results are in a good agreement with previous muffin-tin orbital calculations performed by Yang et al. ${ }^{17}$ showing that metallic character of $\mathrm{LaNiO}_{3}$ is mainly formed by Ni $e_{g}$ subband.

\section{SUMMARY}

To summarize, we have carried out the XPS analysis on $\mathrm{LaNiO}_{3-x}$ thin films deposited onto a monocrystalline (100)plane oriented $\mathrm{NdGaO}_{3}$ substrate. Our theoretical investigations at both crystal and molecular levels are based on LCAO approach enabling to have a better insight on chemi- 


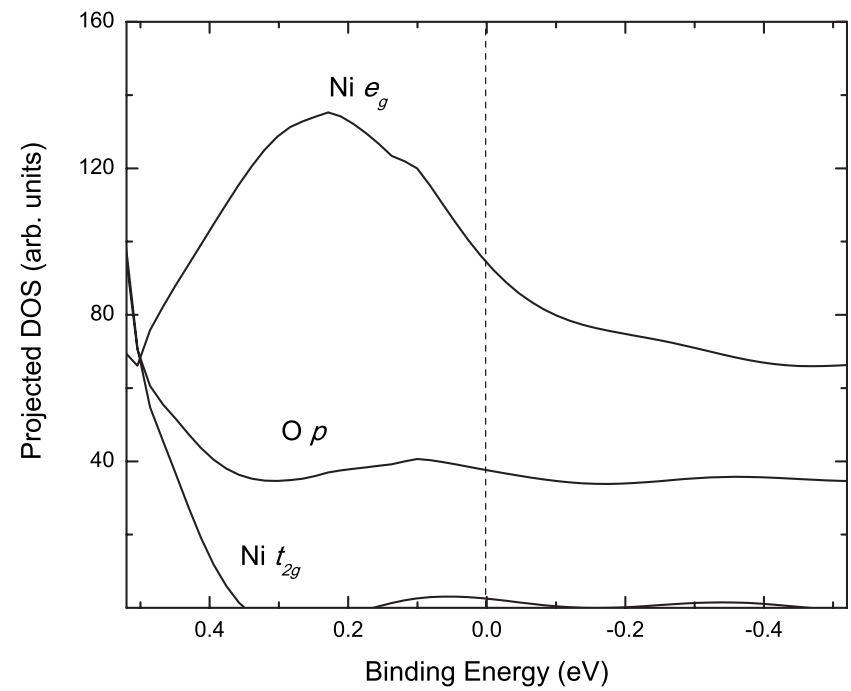

FIG. 9. The projected DOS in the vicinity of the Fermi level calculated using BLYP functional.

cal bonding in $\mathrm{LaNiO}_{3}$ compared to the other basis sets. The crystal calculations performed within hybrid DFT/HF framework shows a good agreement with experimental spectrum in the core region, whereas pure DFT calculations properly represent the observed valence region indicating the strongly delocalized nature of valence electrons. The computationally demanding relativistic molecular study performed within HF framework reveals the importance of spin-orbit interaction to the electronic structure of $\mathrm{LaNiO}_{3}$. As relativistic DOS exhibits a good qualitative agreement with experimental $\mathrm{La}$ $5 p / O 2 s$ spectrum, it allows to theoretically identify the peaks of the doublet splitting of La $5 p$. The band-structure analysis confirms the metallic character of $\mathrm{LaNiO}_{3}$ and the projected DOS in the vicinity of the Fermi level shows that it is formed by dominant Ni $e_{g}$ subband mixed with some part of $\mathrm{O} p$. The electron-density difference map and the hybridization of atomic orbitals imply that covalent contribution to the Ni-O bond is strong while La-O bond is of predominantly ionic nature. The results of the Mulliken population analysis indicate the need of systematic basis set optimization for La.

\section{ACKNOWLEDGMENTS}

This work was partially supported by DESY and the European Commission under Contract No. RII3-CT-2004506008 (IA-SFS) project DESY-D-I-20070119EC, as well as by World Federation of Scientists. Š.M. also acknowledges the Lithuanian Science Council. *sarunas.masys@tfai.vu.lt

†sigism@pfi.lt

†valdas.jonauskas@tfai.vu.lt

${ }^{1}$ J. L. García-Muñoz, J. Rodríguez-Carvajal, P. Lacorre, and J. B. Torrance, Phys. Rev. B 46, 4414 (1992).

${ }^{2}$ D. H. Kuo, C. Y. Chou, and Y. K. Kuo, Int. J. Appl. Ceram. Technol. 7, 217 (2010)

${ }^{3}$ H. N. Aiyer, A. R. Raju, G. N. Subbanna, and C. N. R. Rao, Chem. Mater. 9, 755 (1997).

${ }^{4}$ R. Chiba, F. Yoshimura, and Y. Sakurai, Solid State Ionics 124, 281 (1999).

${ }^{5}$ C. M. Carlson, P. A. Parilla, M. P. Siegal, D. S. Ginley, Y.-T. Wang, R. D. Blaugher, J. C. Price, D. L. Overmyer, and E. L. Venturini, Appl. Phys. Lett. 75, 2479 (1999).

${ }^{6}$ S. S. Kim, B. I. Kim, Y. B. Park, T. S. Kang, and J. H. Je, Appl. Surf. Sci. 169-170, 553 (2001).

${ }^{7}$ J. Choisnet, N. Abadzhieva, P. Stefanov, D. Klissurski, J. M. Bassat, V. Rives, and L. Minchev, J. Chem. Soc., Faraday Trans. 90, 1987 (1994).

${ }^{8}$ S. Mickevicius, S. Grebinskij, V. Bondarenka, V. Lisauskas, K. Šliužienè, H. Tvardauskas, B. Vengalis, B. A. Orlowski, V. Osinniy, and W. Drube, Micron 40, 135 (2009).

${ }^{9}$ P. Chen, S. Y. Xu, W. Z. Zhou, C. K. Ong, and D. F. Cui, J. Appl. Phys. 85, 3000 (1999).

${ }^{10}$ F. Sánchez, C. Ferrater, X. Alcobé, J. Bassas, M. V. GarcíaCuenca, and M. Varela, Thin Solid Films 384, 200 (2001).

${ }^{11}$ Q. He, D. K. Christen, R. Feenstra, D. P. Norton, M. Paranthaman, E. D. Specht, D. F. Lee, A. Goyal, and D. M. Kroeger, Physica C 314, 105 (1999).

${ }^{12}$ K.-S. Hwang, S.-S. Min, and Y.-J. Park, Surf. Coat. Technol.
137, 205 (2001).

${ }^{13}$ V. Lisauskas, B. Vengalis, K. Šliužienė, and V. Pyragas, Phys. Chem. Solid State 9, 350 (2008).

${ }^{14}$ S. Mickevicius, S. Grebinskj, V. Bondarenka, V. Lisauskas, K. Sliuzienè, H. Tvardauskas, B. Vengalis, B. A. Orlowski, V. Osinniy, and W. Drube, Acta Phys. Pol. A 112, 113 (2007).

${ }^{15}$ A. Y. Dobin, K. R. Nikolaev, I. N. Krivorotov, R. M. Wentzcovitch, E. D. Dahlberg, and A. M. Goldman, Phys. Rev. B 68, 113408 (2003).

${ }^{16}$ K. Horiba, R. Eguchi, M. Taguchi, A. Chainani, A. Kikkawa, Y. Senba, H. Ohashi, and S. Shin, Phys. Rev. B 76, 155104 (2007).

${ }^{17}$ Z. Yang, L. Ye, and X. Xie, Phys. Status Solidi B 220, 885 (2000).

${ }^{18}$ Y.-L. Lee, J. Kleis, J. Rossmeisl, and D. Morgan, Phys. Rev. B 80, 224101 (2009).

${ }^{19}$ Y. Nohara, S. Yamamoto, and T. Fujiwara, Phys. Rev. B 79, 195110 (2009).

${ }^{20}$ D. F. Mullica, H. O. Perkins, C. K. C. Lok, and V. Young, J. Electron Spectrosc. Relat. Phenom. 61, 337 (1993).

${ }^{21}$ R. Dovesi, V. R. Saunders, R. Roetti, R. Orlando, C. M. Zicovich-Wilson, F. Pascale, B. Civalleri, K. Doll, N. M. Harrison, I. J. Bush, P. DArco, and M. Llunell, CRYSTAL06 user's manual, 2006.

${ }^{22}$ P. J. Hay and W. R. Wadt, J. Chem. Phys. 82, 299 (1985).

${ }^{23}$ Y.-S. Su, T. A. Kaplan, S. D. Mahanti, and J. F. Harrison, Phys. Rev. B 59, 10521 (1999).

${ }^{24}$ J. K. Perry, J. Tahir-Kheli, and W. A. Goddard, Phys. Rev. B 63, 144510 (2001).

${ }^{25}$ J. Yang and M. Dolg, Theor. Chem. Acc. 113, 212 (2005).

${ }^{26} \mathrm{http} / / /$ www.crystal.unito.it/Basis_Sets/Ptable.html 
${ }^{27}$ M. D. Towler, N. L. Allan, N. M. Harrison, V. R. Saunders, W. C. Mackrodt, and E. Aprà, Phys. Rev. B 50, 5041 (1994).

${ }^{28}$ M. Corno, C. Busco, B. Civalleri, and P. Ugliengo, Phys. Chem. Chem. Phys. 8, 2464 (2006).

${ }^{29}$ A. D. Becke, Phys. Rev. A 38, 3098 (1988).

${ }^{30}$ C. Lee, W. Yang, and R. G. Parr, Phys. Rev. B 37, 785 (1988).

${ }^{31}$ J. P. Perdew, K. Burke, and M. Ernzerhof, Phys. Rev. Lett. 77, 3865 (1996).

${ }^{32}$ A. D. Becke, J. Chem. Phys. 98, 5648 (1993).

${ }^{33}$ C. Adamo and V. Barone, J. Chem. Phys. 110, 6158 (1999).

${ }^{34}$ H. J. Monkhorst and J. D. Pack, Phys. Rev. B 13, 5188 (1976).

${ }^{35}$ D. G. Anderson, J. ACM 12, 547 (1965).

${ }^{36}$ DIRAC, a relativistic $a b$ initio electronic-structure program, Release DIRAC08, 2008, written by L. Visscher, H. J. Aagaard Jensen, and T. Saue, with new contributions from R. Bast, S. Dubillard, K. G. Dyall, U. Ekström, E. Eliav, T. Fleig, A. S. P. Gomes, T. U. Helgaker, J. Henriksson, M. Iliaš, Ch. R. Jacob, S. Knecht, P. Norman, J. Olsen, M. Pernpointner, K. Ruud, P. Sałek, and J. Sikkema, see http://dirac.chem.sdu.dk

${ }^{37}$ M. J. Frisch, G. W. Trucks, H. B. Schlegel, G. E. Scuseria, M. A. Robb, J. R. Cheeseman, J. A. Montgomery, Jr., T. Vreven, K. N. Kudin, J. C. Burant, J. M. Millam, S. S. Iyengar, J. Tomasi, V. Barone, B. Mennucci, M. Cossi, G. Scalmani, N. Rega, G. A. Petersson, H. Nakatsuji, M. Hada, M. Ehara, K. Toyota, R. Fukuda, J. Hasegawa, M. Ishida, T. Nakajima, Y. Honda, O. Kitao, H. Nakai, M. Klene, X. Li, J. E. Knox, H. P. Hratchian, J.
B. Cross, V. Bakken, C. Adamo, J. Jaramillo, R. Gomperts, R. E. Stratmann, O. Yazyev, A. J. Austin, R. Cammi, C. Pomelli, J. W. Ochterski, P. Y. Ayala, K. Morokuma, G. A. Voth, P. Salvador, J. J. Dannenberg, V. G. Zakrzewski, S. Dapprich, A. D. Daniels, M. C. Strain, O. Farkas, D. K. Malick, A. D. Rabuck, K. Raghavachari, J. B. Foresman, J. V. Ortiz, Q. Cui, A. G. Baboul, S. Clifford, J. Cioslowski, B. B. Stefanov, G. Liu, A. Liashenko, P. Piskorz, I. Komaromi, R. L. Martin, D. J. Fox, T. Keith, M. A. Al-Laham, C. Y. Peng, A. Nanayakkara, M. Challacombe, P. M. W. Gill, B. Johnson, W. Chen, M. W. Wong, C. Gonzalez, and J. A. Pople, Gaussian 03, Revision D.01, Gaussian, Inc., Wallingford, CT, 2004.

${ }^{38}$ M. Ilias, H. J. A. Jensen, V. Kellö, B. O. Roos, and M. Urban, Chem. Phys. Lett. 408, 210 (2005).

${ }^{39}$ A. Gomes, K. Dyall, and L. Visscher, Theor. Chem. Acc. (to be published).

${ }^{40}$ K. D. Dobbs and W. J. Hehre, J. Comput. Chem. 8, 861 (1987).

${ }^{41}$ W. J. Hehre, R. Ditchfield, R. F. Stewart, and J. A. Pople, J. Chem. Phys. 52, 2769 (1970).

${ }^{42}$ N. M. O'boyle, A. L. Tenderholt, and K. M. Langner, J. Comput. Chem. 29, 839 (2008).

${ }^{43}$ Photoemission in Solids II, Topics in Applied Physics Vol. 27, edited by M. Cardona and L. Ley (Springer, Berlin, 1979).

${ }^{44}$ R. A. Evarestov, Quantum Chemistry of Solids: The LCAO First Principles Treatment of Crystals (Springer, Berlin, 2007). 